\title{
Using the semantic Web services to build a virtual medical analysis laboratory
}

\author{
Houda El Bouhissi ${ }^{1}$, Mimoun Malki ${ }^{1}$, Djamila Berramdane ${ }^{1}$, Rafa E. Al-Qutaish ${ }^{2}$ \\ ${ }^{1}$ Dept. of Computer Sciences, EEDIS Laboratory, Sidi-Bel-Abbes University, Algeria \\ ${ }^{2}$ Dept. of Software Engineering \& IT, ÉTS, University of Québec, 1100 Notre-Dame West, Montreal, Québec, H3C 1K3, Canada
}

\section{Email address:}

houda.elbouhissi@gmail.com (H. E. Bouhissi),mimoun.malki@gmail.com (M. Malki), berramdane@gmail.com (D. Berramdane), rafa@ieee.org (R. E. Al-Qutaish)

\section{To cite this article:}

Houda El Bouhissi, Mimoun Malki, Djamila Berramdane, Rafa E. Al-Qutaish. Using the Semantic Web Services to Build a Virtual Medical Analysis Laboratory. American Journal of Software Engineering and Applications. Vol. 2, No. 2, 2013; pp. 80-85.

doi: 10.11648/j.ajsea.20130202.17

\begin{abstract}
In medical analysis field, patients often must visit a multitude of laboratories related web sites in order to check availability, booking, prices, result duration, and find the nearest laboratory. Thus, these varieties of reasons to visit the web sites make limitations on the usability of them. However, to overcome these limitations, this paper proposes a Virtual Medical Analysis Laboratory (VMAL) prototype system which will be based on applying the Semantic Web Services (SWSs) for scheduling outpatient tests in order to discover the suitable laboratory. Furthermore, the proposed prototype will also be based on the Web Service Modeling Ontology (WSMO).
\end{abstract}

Keywords: Ontology, Web Services, Semantic Web Services, WSMO

\section{Introduction}

Web Services are software components that are accessible via the Web. However, their concomitant descriptive languages and Web Services Description Language (WSDL) do not offer sufficient semantic richness that can be processed by machine [1]. Furthermore, human intervention is often needed to interpret the meanings in order to discover, compose, and invoke Web services, but this can be time consuming and error-prone [2].

The semantic Web researchers propose many approaches to augment the Web services with a semantic description of their functionality in order to facilitate their discovery and integration [3]. These approaches consist of a combination of Web services with semantic Web technology, and are referred to as Semantic Web Services (SWSs). In addition, the SWSs have the potentiality to modify the knowledge and business services which are given and used on the web [4].

Discovery is one of the central reasoning tasks in Service-Oriented Architecture (SOA) systems, concerned with the detection of usable Web services for a specific request or application context. SWS discovery helps to discover an appropriate service according to user requirements in such scenario. This is the first step towards service selection and composition in order to complete a specific task especially in business environment. Therefore, this paper aims at discovering the suitable SWS taking into account the existing approaches.

In addition, it will propose a prototype system by applying the SWSs for scheduling outpatient tests (blood tests, urine, etc.). Then, this prototype system could be used to discover the suitable laboratory. Also, it will present a state of the art of current enabling SWS technologies and describes the proposed prototype, thus, each Web service has been implemented in a different technologies in order to make them more flexible.

The paper is organized as follows; Section 2 summarizes the main approaches related to SWSs technology. Section 3 introduces the proposed prototype in details. Finally, section 4 concludes the paper and outlines the future work.

\section{Semantic Web Services Approaches}

Web service technologies bring a dynamic aspect to overall Web usage. However, the current understanding about Web services fails to capture enough semantic data. Therefore, semantic services deal with such limitation by augmenting the service description with a semantic layer in order to achieve automated discovery, composition, 
monitoring, and execution, which are all highly desirable processes [5].

However, several approaches have already been suggested for adding semantic to Web services. Semantics can either be added to currently existing syntactic Web service standards such as Universal Description, Discovery and Integration (UDDI) [6] and Web Services Description Language (WSDL), or services can be described using some ontology based description language. The Major initiatives in the area of SWSs are documented by W3C member submissions, such as, OWL-S [7], WSMO [8] and WSDL-S [9].

Ontology Web Language for Services (OWL-S) is a description language that semantically describes Web Services using OWL ontologies. OWL-S services are mapped to WSDL operations, and inputs and outputs of OWL-S are mapped to WSDL messages.

The Web Services Description Language - Semantic (WSDL-S) is an evolutionary and backwards compatible extension of the existing Web Services standards and descriptions language, which augments the expressivity of
WSDL with semantics in an arbitrary semantic representation language. In addition, it provides a means to supply semantic information, but actual semantic functionality has to be provided by additional components, which are not part of the WSDL-S initiative.

The WSDL-S proposal was replaced by Semantic Annotations for WSDL (SAWSDL) [10], a W3C recommendation, which is a simple and generic mechanism for semantically annotating Web Service descriptions. The SAWSDL is a restricted and homogenized version of WSDL-S in which annotations like preconditions and effects have not been explicitly contemplated.

The Web Services Modeling Ontology (WSMO) provides ontological specifications for the description of SWSs [11]. One of the main objectives of WSMO is to give a solution to application integration problems for Web Services by providing a conceptual framework and a formal language for semantically describing all relevant aspects of Web Services [12], Table 1 depicts the main differences between the three approaches.

Table 1. Comparison between the three approaches

\begin{tabular}{llll}
\hline & OWL-S & WSMO & WSDL-S \\
\hline Scope & $\begin{array}{l}\text { Description model for } \\
\text { semantically describing Web }\end{array}$ & $\begin{array}{l}\text { Description model \& language for core } \\
\text { elements of Semantic Web Service }\end{array}$ & $\begin{array}{l}\text { Semantic Annotation of } \\
\text { technologies }\end{array}$ \\
& Services & Ontologies & \\
Top Level Elements & Service Profile & Goals & Operations/ \\
& Process model & Web Services & WSDL descriptions \\
& Grounding & Mediators & \\
Language & OWL & WSML & Not specified \\
Maturity of the approach & Strong & Medium & Medium \\
Mediation & Under development & Strong (MEDIATORS elements) & Not specified \\
\hline
\end{tabular}

WSMO is the only initiative which has an explicit notion of mediation [13]. Furthermore, WSMO is the only standard for which there exist several implementation environments which aim to support the complete standard [14]. For these reasons WSMO is used as our SWSs technology throughout the rest of this paper. In addition, WSMO model consists of both the requester and provider sides, and thus, it gives the opportunity to the requester (user) to define a goal in order to find a semantically annotated web service.

Ait-Ameur [15] proposes a semantic repository for adaptive services in which he uses a semantic registry, to store SWSs. However, this, semantic registry is equipped with an exploitation language that supports semantic based process discovery. Moreover, Belaid et al. [16] apply ontology and indexation based management of services and workflows to the Geological Modeling.

\section{The Proposed Prototype}

\subsection{Motivation}

It is clear that finding out the interested services without using automated mechanisms is very difficult and time consuming. This issue is similar to search in web pages without using browsers. Service consumer can be user, another service or a program. Thus, using of automated mechanisms to finding out services is very important [17].

SWSs technology can be used to optimize several processes in the domain of e-health, especially in Medical Analysis [18]. These processes are mainly related to human interactions, and consequently to the costs associated with them. Hence, the main benefit of applying SWS technology is that it could permit to develop and maintain e-health services with lower costs.

The use of SWSs technology can optimize this manual process by allowing a search in available registries, so that the new Web Services which have been deployed can be discovered easily and quickly.

Figure 1 below, presents two scenarios that motivated us to develop this prototype. In the first scenario (Scenario 1), for an outpatient test (blood test, urine test, etc.), the patient often must visit the web sites for many different 
laboratories in order to check availability, prices, result duration and also to find the nearest laboratory.

Using phone directories is also needed if some web sites' laboratories are not available. The patient should contact each laboratory by phone or email to get the necessary information, such as, address, distance from home, price, possible results, etc. This method is very difficult and time consumer, especially when the patient needs to visit many laboratories.

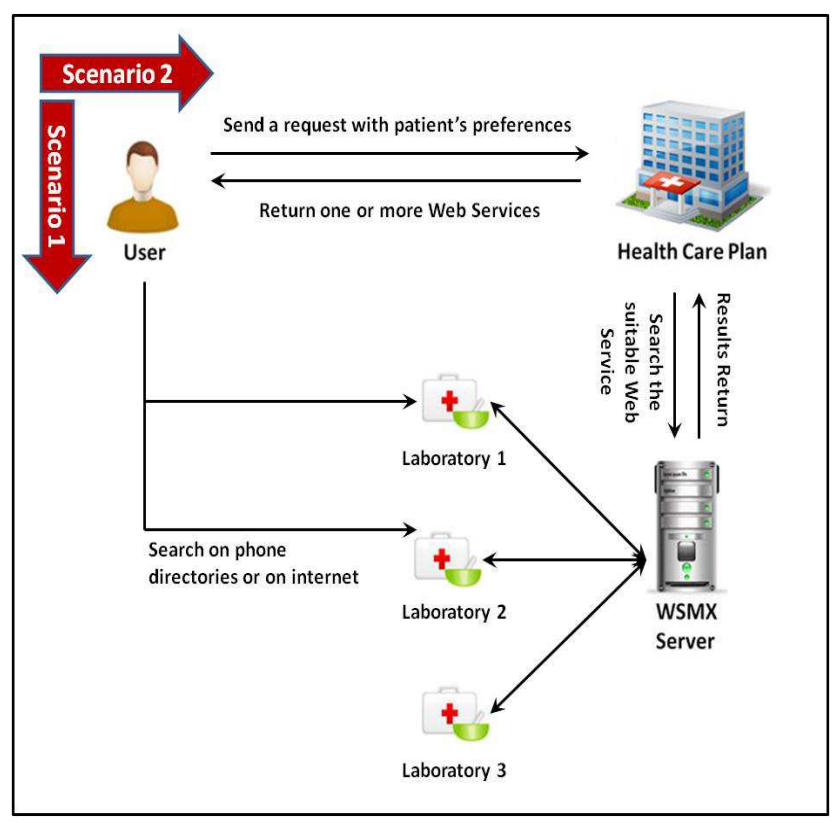

Figure 1. The Motivation Scenario

In the second scenario (Scenario 2), to overcome the limitations in the first scenario, the Virtual Medical Analysis Laboratory (VMAL) prototype system is proposed to discover the suitable Laboratory by applying SWSs for scheduling outpatient tests (blood tests, urine tests, etc.).

In order to model the scenario, we propose prototype based on Web service Modeling Ontology (WSMO), we use WSMO for modeling of services and goals (i.e. required and offered capabilities) as well as ontologies (i.e. information models on which services and goals are defined) all written in the WSML ontology language.

The scheduling of the required tests could be done through the following steps:

- The patient informs what tests she/he wants to do (blood test, urine test, etc.);

- The system discovers what laboratories can do these tests (discover web services from laboratories); and

- The system returns a list of one or more discovered Web Services to patient.

As shown in Figure 1, the patient sends a request to the portal with her/his preferences. A searching process for suitable Web Services is performed in the WSMX server where all the Web Services are stored. At last, the patient receives one or more Web Services according to her/his request. Next subsections will explain in more details the main architecture of the prototype.

\subsection{The WSMO Framework}

WSMO (Web service Modeling Ontology) is a formal ontology and language which identifies the following four main top-elements:

- Ontologies that provide the terminology used by other elements;

- Goals that state the intentions which should be solved by Web Services;

- Web Services descriptions which describe various aspects of a service; and

- Mediators to resolve interoperability problems.

Each of these WSMO Top Level Elements can be described with non-functional properties, such as, creator, creation date, format, language, owner, rights, source, type, etc. Furthermore, WSMO comes along with a modeling language (WSML) and a reference implementation (WSMX).

The WSML (Web Service Modeling Language) is a formalization of the WSMO ontology and providing a language within which the properties of SWSs can be described.

Whereas, the WSMX (Web Service eXecution Environment) provides an architecture including discovery, mediation, selection, and invocation. In addition, it has been designed to include all the required supporting components enabling an exchange of messages between requesters and the providers of services.

\subsection{Architecture of the Prototype}

The VMAL prototype could be used to help users of the medical laboratories to schedule their outpatient tests (blood tests, urine tests, etc.)

Figure 2 presents the architecture of the prototype. The patient communicates with the VLMA portal via the HTTPS protocol, which provides a secure communication channel.

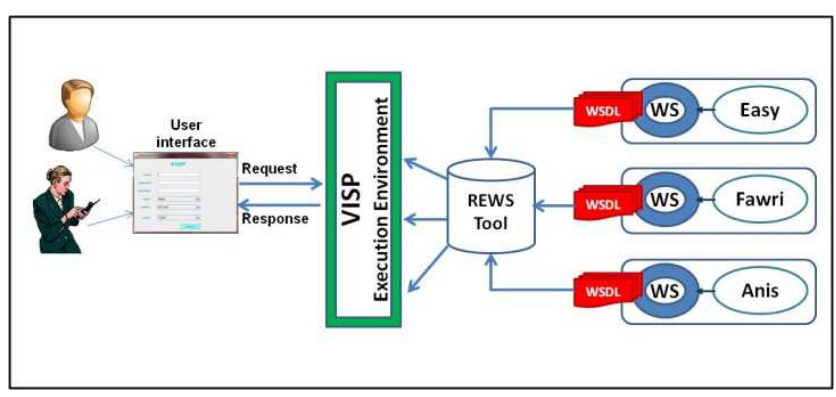

Figure 2. The Architecture of the prototype

The essential functionalities of the proposed prototype process applied to medical analysis are as the following:

- Provide a friendly interface for patient interaction.

- Discover suitable Web Services according to patient preferences (availability, distance, price, etc.).

Much of the promise of Web Services is its potential for seamless interoperability across heterogeneous systems, platforms, applications, and programming languages. Interoperability is a primary goal of web services. Web 
services standards facilitate interoperability, but do not ensure it.

The proposed prototype consists of three Web Services, a WSMX server and a simple interface client application implemented with Java language that communicates with the WSMX Server. The three Web Services are implemented with different platforms and programming languages. The use of several programming languages makes our prototype more flexible because we consider that the WSDL file as our only source of information and the choice of these programming languages is random. The first Web Service was implemented with the Java language and the NetBeans Platform, the second with the .NET, and the third one was implemented with PHP and MYSQL.

Service providers publish their service and made a description of it in Web Service Description Language (WSDL). WSDL provides the way through which Web Services can be described according to their functional, non-functional behavior.

All the WSDL files are submitted to the Reverse Engineering Web Service Application (REWS) tool [19] for extracting the main WSMO Web Service elements, which are written in WSML language and then will be stored in the WSMX Server.

In our prototype, the WSMX server plays the role of a UDDI directory where all Web Services elements are stored.

All semantic descriptions are provided on top of existing providers' syntactic services, making providers unaware of this semantic layer. No changes are involved in providers' services and native data formats are preserved.

The WSMX server is a computer where the WSMX is installed and configured, it acts as a transparent and intermediary layer between interacting parties for mapping and discovery. It consists of some components that fulfill Web Services tasks such as discovery, invocation, composition, etc.

The patients' desires are expressed via web forms (see Figure 3) which are mapped to appropriate Goal and expressed in WSML language.
Once a WSMO Goal with its actual values is created, then it can be sent to WSMX, where provider matching this Goal is discovered, and according to Goal and Web service choreography communication is carried out. At last, one or more Web Services are returned to the patient.

This scenario is very simple, and the use of SWS is needed in this case. The reason for choosing this scenario is to keep the implementation within the scope of the project and because it uses the most important and basic parts of WSMX.

To be able to fulfill this scenario, WSMO ontology elements must be created to provide a shared vocabulary for the different interactions.

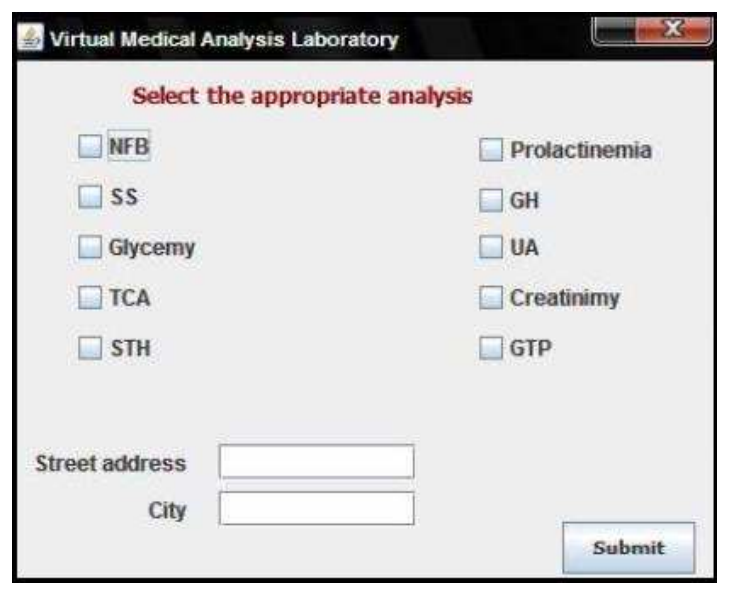

Figure 3. User interface snapshot

\subsection{System Phases}

According to WSMO, to develop SWS, the actual Web Service should be implemented; a WSMO Web Service should be created to describe the web service. Also, the necessary ontologies used by the Web Service should be defined. Then, to test it, a goal needs to be created, that is, to represent a request to WSMX and the ontologies used by it. The discovery process can be split into eight high-level steps, see Figure 4.

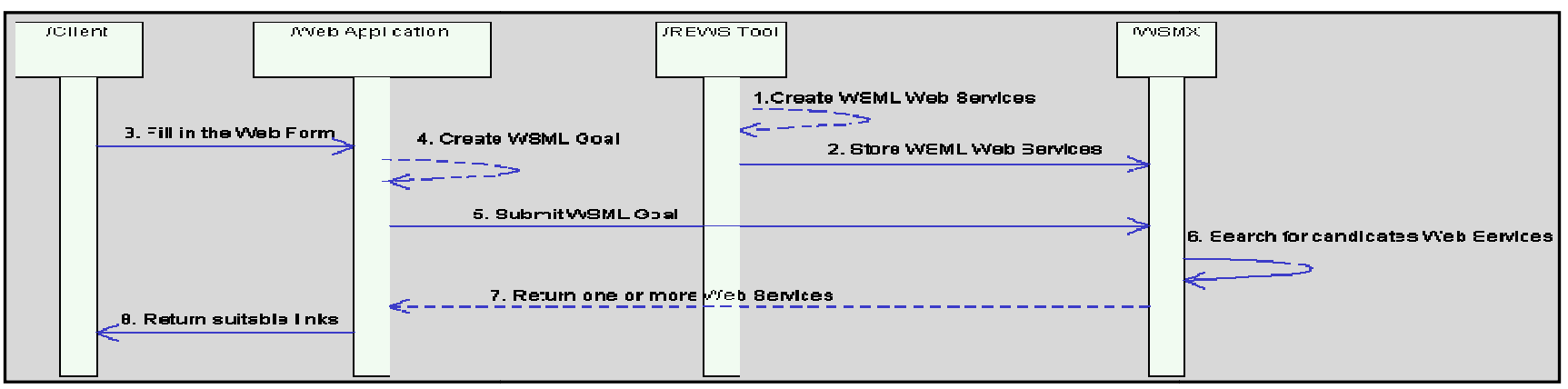

Figure 4. Sequence diagram for discovery of Web Services

The following describes, in more details, the different phases of the process.

\section{Phase 1: Creating WSMO Web Services Elements}

Providers' Web Services have to be semantically described to include lifting arbitrary XML messages in WSDL document to the semantic level by the ontology conceptualization. 
In this phase, the REWS tool has been used to create WSMO Web Services elements. It takes as input WSDL File of each Web Service and generates as output the web Service Elements expressed in WSML language. Then, these elements will be stored on the WSMX server for further using. Figure 5, depicts part of a WSML file according to the first Web Service.

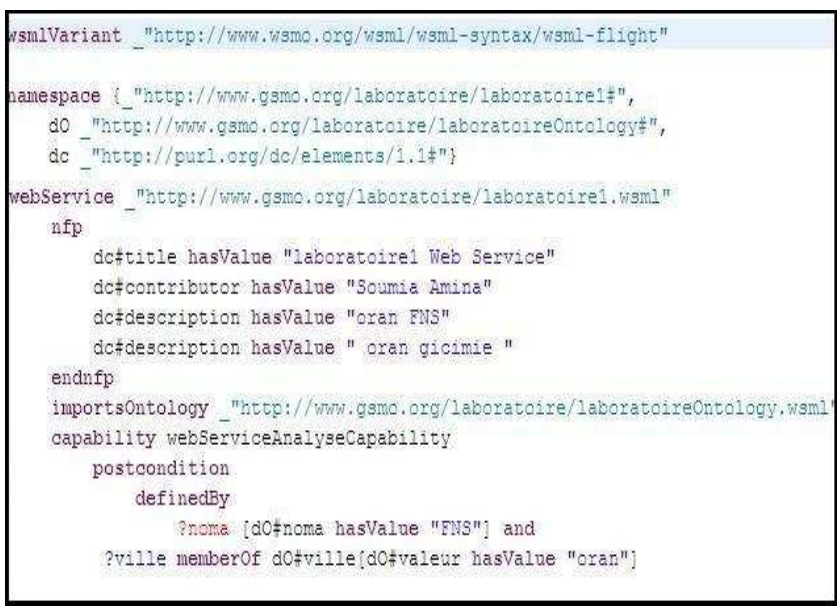

Figure 5. Snapshot of a part of WML Web Service file

\section{Phase 2: Creating WSMO Goals}

Patient does not have to visit multiple web sites, but can use only the friendly interface provided by the portal (see Figure 3 above) that aggregates multiple Medical analysis services, and sure it can be extended with new ones.

The requirements and behavior of the client have to be expressed as WSMO Goal. A Web application implemented with Java and NetBeans provides forms where user can specify her/his requirements and input values.

The patient has to introduce necessary data to find Medical analysis offers.

The system accept the expression of patients' goals using Web forms that in turn are mapped to WSMO Goals, and allow them to be executed by WSMX.

In the proposed prototype, the Goals are based on a template approach where the Goal structure is defined but actual input values can be provided during the run-time by the patient. Also we create an ontology for such goal(see figure 6).

\section{Phase 3: Matching Web Service to Goal}

Now, the Goal is submitted to WSMX, whereas, the provider matching this Goal is discovered, and then according to the Goal itself and Web Service choreography communication is carried out [20].

The Discovery component of the WSMX server then starts to match the capabilities required by the goal, with the capabilities of the Web Services already registered in WSMX.

The Service Discovery component refines the suggested Web Services resulted from the discovery and chooses the most appropriate web service based on the requester's preferences

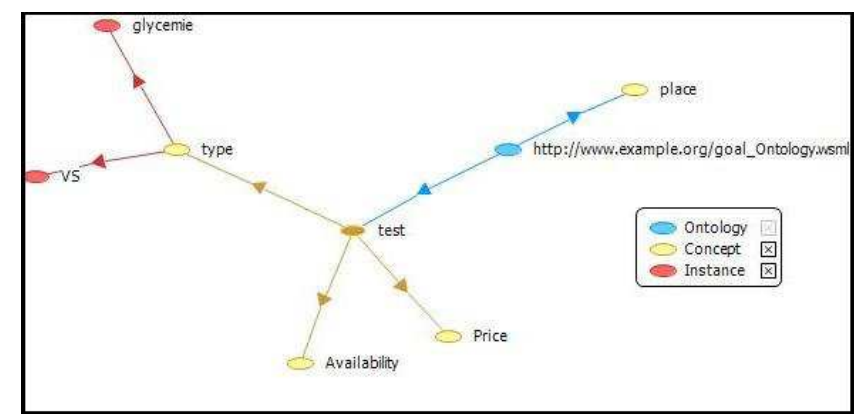

Figure 6. Ontology according to WSMO goal

This matching is performed by the discovery component of the WSMX platform and it can be held using different criteria that are further discussed by [21]. The default matching criteria are the Keyword-based discovery and the LightWeight discovery.

The former relies on matching the Non-Functional properties defined by the Web Service and the goal, while the latter compares the Postconditions and the Effects specified in the two interfaces.

After that the Service Discovery component refines the suggested web services resulted from the discovery and chooses the most appropriate web service based on the requester's preferences. Some user preferences can be specified using Non-Functional properties.

After the web service is chosen, a response is returned to the patient with suitable Web Services links.

WSMX takes a semi-automatic approach to this problem. Initially, three Web Services were loaded to WSMX. Then to perform the discovery, the WSMX server used both the Keyword-based and the LightWeight discoveries. Two Web Services were discovered, the first one using the Keyword discovery and the second one using the LightWeight (see figure 7 below).

The human's role is to ensure accuracy of these mappings and to adjust them if necessary.

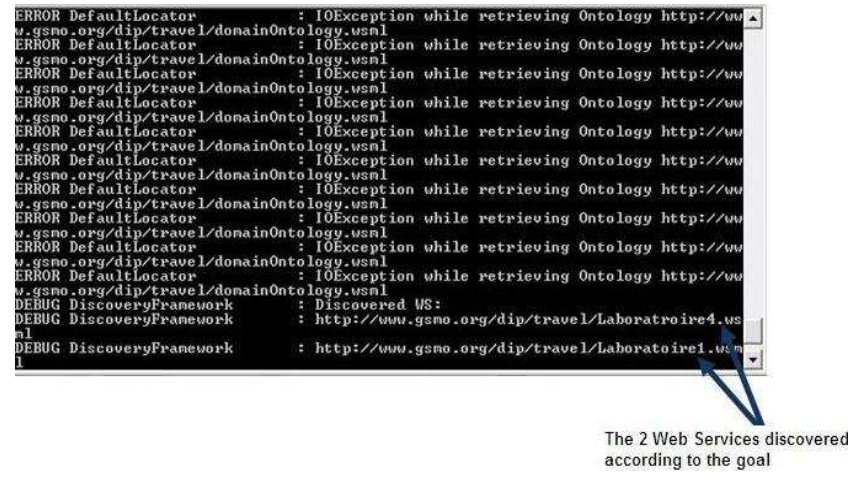

Figure 7. Snapshot of the discovered Web Services

\section{Conclusion}

SWSs are very powerful paradigm which could be used to transform the current syntactic Web into a dynamic and semantic one, thus, this transformation allows automating the use of the Web Services. 
Especially, the discovery of SWSs has become an important when large number services are available for use on the Internet, so the acquisition of appropriate SWS is the main goal for a user who is searching for a service.

This paper describes a prototype of a Virtual Medical Analysis Laboratory (VMAL) application for demonstrating how the application of SWSs technology makes it possible for individual patients to find the suitable laboratory for scheduling outpatient tests.

As a future work, this proposed prototype could be extended to transport Web Services. In addition, such prototype could also be used with many applications in Web Services.

\section{References}

[1] W3C, Web Services Description Language (WSDL) 1.1, available online at: http://www/w3.org/TR/wsdl, retrieved on: March 7, 2013.

[2] L. A. Kamaruddin, J. Shen and G. Beydoun, Evaluating Usage of WSMO and OWL-S in Semantic Web Services, In Proceedings of the 8th Asia-Pacific Conference on Conceptual Modelling (APCCM'12), RMIT, Melbourne, Australian, January 30 - February 2, 2012, pp. 53-58.

[3] A. Suganthy, G. S. Sumithra, J. Hindusha, A. Gayathri and S. Girija, Semantic Web Services and its Challenges, International Journal of Computer Engineering and Technology (IJCET), Vol. 1, No. 2, 2010, pp. .26-37.

[4] L. Cabral, J. Domingue, E. Motta, T. Payne and F. Hakimpour, Approaches to Semantic Web Services: An Overview and Comparisons, In Proceedings of the 1st European Semantic Web Symposium, Heraklion, Greece, May 10-12, 2004.

[5] Antoniou, G.; van Harmelen, F., 2008. A Semantic Web Primer. The MIH Press, Cambridge, USA.

[6] OASIS, UDDI Version 3.0.2, UDDI Spec Technical Committee Draft, available online at: http://uddi.org/pubs /uddi_v3.htm, retrieved on: March 7, 2013.

[7] D. Martin, M. Burstein, J. Hobbs, O. Lassila, D.McDermott, S.McIlraith, S.Narayanan, M.Paolucci, B. Parsia, T. Payne, E.Sirin, N.Srinivasan and K. Sycara, OWL Web Ontology Language for Services $(O W L-S)$, available online at: http://www.w3.org/Submission/OWL-S, retrieved on: March 7, 2013.

[8] J.d. Bruijn, C. Bussler, J. Domingue, D. Fensel, M. Hepp, U. Keller, M. Kifer, B. König-Ries, J. Kopecky, R. Lara, H. Lausen, E. Oren, A. Polleres, D. Roman, J. Scicluna and M. Stollberg, Web Service Modeling Ontology (WSMO), available online at: http://www.w3.org/Submission/WSMO, retrieved on: March 7, 2013.

[9] R. E. Akkiraju, J. Farrell, J. Miller, M. Nagarajan M. Schmidt, A. Sheth and K. Verma, Web Service Semantics - WSDL-S,
W3C Member Submission 7, 2005, available online at: http://www.w3.org/Submission/2005/SUBM-WSDL-S-2005 1107, retrieved on: March 7, 2013.

[10] J. Farrell and H. Lausen (eds), Semantic Annotations for WSDL and XML Schema. W3C Candidate Recommendation, available online at: http://www.w3.org/TR/sawsdl, retrieved on: March 7, 2013.

[11] H. El Bouhissi and M. Malki and D. Bouchiha, Towards WSMO Ontology Specification From Existing Web Services, In Proceedings of 2 nd Conférence Internationale sur l'Informatique et ses Applications (CIIA 2009), Saida, Algeria, May 3-4, 2009.

[12] F. Cristina and J. Domingue, D2v1.0 WSMO Primer: CMS WG Working Draft, available online at: http://cms-wg. sti2.org/TR/d2/v1.0/20050401, 2005, retrieved on: March 7, 2013.

[13] ESSI WSML working group, Web Service Modeling Language WSML, available online at: http://www. wsmo.org/wsml, retrieved on: March 7, 2013.

[14] Web Service Execution Environment, Web Service Modeling Ontology, available online at: http://www.wsmx.org, retrieved on: March 7, 2013.

[15] Y. Ait-Ameur, A Semantic Repository for Adaptive Services, In Proceedings of the IEEE Congress on Services (SERVICES'09), Los Angeles, CA, USA, July 6-10, 2009, pp. 211-218.

[16] N. Belaid, S. Jean, Y. Ait-Ameur, J.-F. Rainaud, An Ontology and Indexation Based Management of Services and WorkFlows: Application to Geological Modeling, International Journal of Electronic Business Management (IJEBM), Vol. 9, No. 4, 2011, pp. 296-309.

[17] K. Zamanifar, A. Zohali and N. Nematbakhsh, Matching Model for Semantic Web Services Discovery, In Proceedings of the International Conference on Advanced Information Networking and Applications Workshops, Bradford, United Kingdom, May 26-29, 2009.

[18] L. J. Cox, Feature-Oriented Domain Analysis for Semantic Web Services, M.Sc. Thesis, Athabasca University, Athabasca, Alberta, Canada, December 2010.

[19] H. El Bouhissi and M. Malki, Reverse Engineering Existing Web Service Applications, In Proceeding of the 16th Working Conference on Reverse Engineering (WCRE'09), Lille, France, October 13-16, 2009, pp. 279-283.

[20] H. El Bouhissi and M. Malki, Semantic Web Services: WSMO Goal Based Architecture, In Proceedings of the International Conference on Information and Communication Systems (ICICS'11), Irbid, Jordan, May 22-24, 2011.

[21] M. Herold, Evaluation and Advancement in Context of a Tourist Information System. http://www.fhwedel.de/ fileadmin/mitarbeiter/iw/Abschlussarbeiten/Masterarbeit Herold.pdf, 2008. Retrieved on March 7, 2013. 